\title{
Criterios de pertinencia atribuibles a los actos pedagógicos en la enseñanza de una segunda lengua
}

\author{
Concha Sanz
}

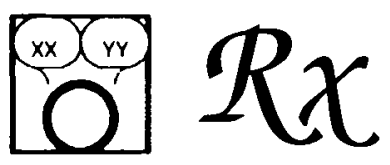

Las posiciones de Vigotski sobre las relaciones entre aprendizaje y desarrollo ban tenido distintas aplicaciones en el ámbito de la enseñanza de las ciencias y las matemáticas, pero son poco conocidas en relación a la enseñanza de la lengua. La autora de este artículo propone, basándose en concepciones vigotskianas, una metodología concreta para enseñar la lengua extranjera.

Implícita o explícitamente, aún persiste en la pedagogía de las lenguas la distinción de facto entre el desarrollo de la lengua materna (L1) como un proceso natural, es decir inconsciente, autogenerado y susceptible de una explicación fundamentalmente biológica, y el de una segunda lengua (L2) como radicalmente cultural, entiéndase como estrictamente racional, consciente y que pasa inevitablemente por un intrincado y dilatado plan de organización del conocimiento, de carácter intrapersonal aunque sabia y rigurosamente dirigido.

Por el contrario, creemos que resultan infrecuentes las experiencias pedagógicas - como la que procedemos a comentar - que conciben de modo consecuente como básica y cargada de consecuencias para la práctica educativa la coincidencia de un conjunto interactivo de factores determinantes del aprendizaje para los dos procesos considerados. Resumimos esos factores del siguiente modo:

1. Por una parte, en palabras de Vygotski, "los procesos de asimilación de la lengua materna y de un idioma extranjero tienen tanto en comin que en esencia se refieren a una clase única de procesos de desarrollo del lenguaje...", (Vygotski, Obras Escogidas II, Visor/Aprendizaje, Madrid, 1993. p. 197), es decir que lo que en definitiva hacemos al aprender una segunda lengua es seguir explorando el potencial de la capacidad privativa de la especie humana que es el lenguaje verbal, o simplemente seguir aprendiendo a hablar.

2. El segundo factor equiparable de carácter fundamental para los dos procesos es una situación permanente de interacción social cuya dinámica crea las condiciones merced a las cuales los objetos de aprendizaje aparecen como necesarios, y el mismo acto de aprendizaje se presenta como natural además de inevitable. 
Este segundo factor no fue tenido en cuenta por la didáctica convencional, ni por cierto por el propio Vygotski quien únicamente tendría la oportunidad de observar experiencias pedagógicas en lenguas extranjeras según el método tradicional de gramática-traducción. Consecuencia de ello son las descripciones de Vygotski del siguiente orden: "Si el desarrollo de la lengua materna parte de su empleo fácil y espontáneo y culmina con la toma de conciencia de las formas verbales y con el dominio de las mismas, en cambio, el desarrollo del idioma extranjero comienza por la toma de conciencia del mismo y por su dominio voluntario y culmina con el lenguaje suelto y espontáneo.» (p. 256).

El estilo de intervención convencional consistente en hacer pasar todas y cada una de las nuevas adquisiciones de la L2 por el tamiz de una toma de conciencia escrupulosamente dirigida, hasta alcanzar la tierra prometida que supondría la automatización de la expresión, no se verifica en la práctica como todo el mundo sabe. La realidad es que tras muchos años de instrucción, sólo un número muy reducido de alumnos alcanzan unos niveles de dominio operativo mínimamente aceptables mediante ese procedimiento, y lo hacen debido, sin duda, a una intuición linguística y una capacidad deductiva excepcionales. Bien es verdad que los logros en cuanto a intelectualización del funcionamiento de la lengua pueden considerarse estimables en esos casos, pero los objetivos declarados no apuntan precisamente en esa dirección, sino al parecer a la capacidad de comunicación.

3. Ligado al segundo factor coincidente para ambos procesos que aludía a la inmersión en una situación de intercomunicación, aparece el tercero, que se refiere a que el desarrollo de la L1, lo mismo que el de la L2, conlleva necesariamente y también de modo permanente, tanto en contexto formal como informal, una relación pedagógica sostenida con las personas del entorno, pues en ambos casos los mayores o los más capaces se esfuerzan en sincronizar su competencia con la del aprendiz del lenguaje, haciendo posible los consabidos dispositivos de andamiaje que ayudan al sujeto a operativizar sus nuevas adquisiciones.

4. En cuarto y último lugar, en concomitancia con los citados procesos dirigidos vienen a confabularse el instinto y el razonamiento espontáneo que a veces producen aprehensiones automáticas del saber, casi por simple percepción, y a veces generan progreso mediante un tanteo experimental más o menos asistido.

Conviene precisar muy resumidamente que el principio metodológico general por el que optamos para la enseñanza de una L2 consiste básicamente en someter al alumno desde el primer día de clase a un ejercicio continuado de verbalización del pensamiento propio, involucrándolo en actividades resolutivas y alternando permanentemente la práctica de la lengua oral y del escrito.

Dicho esto, hay que aclarar también que pese a que pueda parecer que el presente análisis no se detiene suficientemente sobre esta cuestión, no minimizamos ni hacemos caso omiso naturalmente de los determinantes psicológicos que se derivan de la disparidad entre las edades del sujeto en los momentos en que los procesos de desarrollo de la L1 y la L2 se inician. Las consecuencias más destacables son que a mayor edad hallamos para ambos procesos unos niveles de conciencia de la lengua más elevados y que, ciertamente, el techo cuantitativo y cualitativo del tramo más fecundo del proceso de aprendizaje se encuentra significativamente más bajo en el caso de la L2, si bien nuestro criterio al respecto es que el hecho es fundamentalmente tributario de la circunstancia del menor tiempo de exposición al objeto de aprendizaje. Pasada esa etapa gloriosa, el potencial de desarrollo puede considerarse igualmente ilimitado tanto en un caso como en el otro.

Por tanto, no reducimos el factor edad a una variable puramente anecdótica, sin embargo su relevancia indiscutible no tiene una incidencia específica sobre nuestra teoría, por lo que no cuestiona la convicción avalada por nuestra experiencia de que una estrategia educativa que se construye sobre una concepción del proceso de adquisición de 
la L2 basada en el paralelismo fundamental del conjunto de los principales elementos determinantes del aprendizaje con los del proceso de desarrollo de la L1 (origen biológico común-interacción social-procesos dirigidos y espontáneos) resulta mucho más eficaz y nos atreveríamos a añadir que incontestablemente más vygotskiana, como intentaremos probar.

De modo que descartamos por una parte la hipótesis convencional de que el mecanismo de adquisición de una L2 pueda revestir un carácter profundamente específico y novedoso para el proceso evolutivo, como pudiera corresponder al aprendizaje de las matemáticas, por ejemplo. Obviamente, hay que eliminar igualmente la posibilidad de que dicho proceso pueda asemejarse al aprendizaje de las materias consideradas de cultura general como la historia o las ciencias naturales. Por el contrario, asumimos la conclusión de Vygotski según la cual el proceso de adquisición de una $\mathrm{L} 2$ constituye una variante del proceso general de desarrollo del lenguaje de un modo equivalente — probablemente más próximo aún-a lo que en su momento supuso el aprendizaje de la escritura para el niño. Finalmente, añadimos el presupuesto de que la situación de aprendizaje de una L2 puede y debe desarrollarse en circunstancias de interarción social equiparables a las de la L1 si se desea realmente alcanzar cotas aceptables de automatización de la expresión en un plazo evidentemente también prudencial.

Asentados esos principios, lo que procedemos a dilucidar seguidamente es cómo, desde nuestro modelo de intervención, se entiende que podría impulsarse una integración fructífera de los procesos psicológicos autónomos y dirigidos, teniendo en cuenta el impacto respectivo de las dos estrategias pedagógicas contrapuestas por la distinta carga de directividad que encierran.

Rechazamos de plano por supuesto las prácticas relativamente modernas, aunque prácticamente generalizadas en los libros de texto y las aulas, consistentes en efectuar una yuxtaposición mecánica y aleatoria de estrategias directivas y estrategias que promueven las producciones lingüísticas autónomas (media hora —o dos meses - de demostración-/media hora —o cinco minutos- de creatividad) ya que, por una parte, esta forma de proceder no asegura obviamente la interacción necesaria entre las mencionadas estrategias y, por otra, parece presuponer que no existe una base racional sobre la que asentar las decisiones relativas a una distribución fundamentada de las funciones de control entre los agentes del acto educativo: profesor y alumnos.

Nuestra perspectiva permite un enfoque muy distinto y se centra básicamente en procurar compatjbilizar dos ideas fundamentales, ambas con una doble vertiente, cuya observancia permite, a nuestro entender, optimizar las consecuencias de la intervención:

1. La primera reproduce la lógica de la ZDP, y consiste en procurar que el escenario de la intervención permanezca dentro de los márgenes que impone el esfuerzo para evitar por un lado confiar a estrategias directivas los segmentos del acto de aprendizaje que pueden resolverse mediante una actividad autoestructurante, y evitar por otro lado solicitar de los recursos autónomos del alumno realizaciones que solamente cabría desarrollar en condiciones de directividad.

2. Nuestra segunda preocupación guarda un paralelismo evidente con la primera y ofrece tintes igualmente vygotskianos: consiste en procurar permanecer dentro de los márgenes que señala el propósito de evitar recurrir a la toma de conciencia con la pretensión de precipitar aprendizajes lingüísticos que podrían resolverse más eficazmente por aprehensión espontánea o acumulación de experiencia, y no dudar en cambio en recurrir a la misma para hacer frente a las dificultades mayores que manifiestamente sólo pueden ser superadas mediante ese procedimiento.

Todos los segmentos del acto pedagógico que cumplen con las citadas condiciones son considerados como pertinentes. Dicho de otro modo, establecemos que un 
segmento es pertinente en la medida en que el alumno recibe cuando lo necesita, ni más ni menos que el tipo de ayuda que necesita, teoría que subrayamos como prerrequisito insoslayable de una práctica virtualmente exitosa.

Para proceder a la determinación de pertinencia de los segmentos pedagógicos precisamos distinguir tres tipos de actos de aprendizaje habida cuenta del grado de dificultad que impone su consumación.

En el grado 1, incluimos todos los aprendizajes que entrañan una dificultad menor, por ejemplo todo aquello que el alumno domina ya en la L1 y traslada automática y acertadamente al uso de la L2, y en general todos los aprendizajes lingüísticos o no lingüísticos que son asimilados prácticamente sin necesidad de ayuda, sea instantáneamente, a través de la reflexión o autocorrección instintivas, o de la búsqueda espontánea, ágil y diligente de información.

En el grado 2 catalogamos los aprendizajes que requieren que dos estrategias pedagógicas, los recursos autónomos de adquisición y la contribución de otros a iniciativa de otros, se conciten en sincronía o breve alternancia y en una medida equiparable, según un sistema de vasos comunicantes. Se trata por ejemplo de los casos en que una ayuda relativa u orientación circunstancial por parte del profesor o de los compañeros de clase, y/o una guía ocasional y metódica del material pedagógico resultan determinantes para que una actividad autoestructurante pueda proseguir.

Finalmente, incluímos en el grado 3 las dificultades mayores cuya superación resulta imprescindible para el dominio operativo de la lengua o el logro de otras metas formativas marcadas, y que manifiestamente no pueden resolverse a no ser mediante un tipo de intervención en el que predominan las estrategias directivas. En este apartado aparecen dificultades recalcitrantes, errores contumaces, así como tareas resolutivas ambiciosas que exigen o una dedicación complementaria, o un entrenamiento sistemático, o un estudio dirigido, o la realización de ejercicios. Se trata en todo caso de actividades que se apoyan ineludiblemente en el control externo y sostenido de la conciencia.

Tras efectuar este ordenamiento, nos propusimos el doble objetivo de evitar por un lado que el planteamiento teórico que habíamos elaborado (que en realidad procedía a su vez de un deseo de dotar de un mayor nivel de racionalización a unas prácticas ya muy encauzadas desde el punto de vista formal y filosófico) perdiera coherencia en su traslado al campo experiencial y, por otro, evitar igualmente el riesgo evidente de llegar al extremo de someter a los profesores a las tensiones propias de un ejercicio constante y extraordinariamente complicado de dilucidación e improvisación.

La respuesta clásica — no por arcaica menos extendida - a la pregunta de cómo y cuándo debería la intervención dar prioridad a estrategias directivas o, por el contrario, optar preferentemente por favorecer una actividad autoestructurante en la enseñanza de una L2 es muy simple, como ya aludimos más arriba: no se contempla la existencia de una precompetencia en el alumno sino que los recursos operativos autónomos son el presunto producto lógico, la consecuencia automática de la eficacia de un control externo exhaustivo y global de los procesos internos. Por eso en la práctica docente las dos estrategias de adquisición contrapuestas se suceden siempre linealmente en el tiempo. Nos encontramos ante un acto de fe más que de una alternativa razonada: un conocimiento adecuadamente transmitido será convenientemente asimilado (y evaluado), y habrá de mutarse por su propia excelencia en capacidad de expresión (que no se evalúa).

La realidad es que la verdadera consecuencia del sometimiento general y sistemático de los mecanismos psicológicos de aprendizaje a los procesos directivos, el recurso indiscriminado a la manipulación consciente de la L2, y la consideración de los procesos autónomos como un enigma inextricable que más valdría ni abordar, es la 
creación en el alumno de un estado de adicción al sistema, es decir, de consolidación de unas relaciones de dependencia operativa que no hacen más que aplazar, en una proporción equivalente, las oportunidades de desarrollo del potencial operativo autónomo y, en definitiva, de la automatización de la expresión que encuentra siempre su aliado predilecto en la actividad autoestructurante.

Nuestra teoría de la pertinencia del acto pedagógico pretende facilitar la superación de esa simplificación distorsionante de los procesos espontáneos de construcción del conocimiento y perjudicial por tanto para las finalidades de la formación lingüística.

Siguiendo con nuestro razonamiento, llegamos a la conclusión de que la coherencia a los principios marcados exigía que el modelo de intervención consecuente señalara unos ciclos para el comportamiento pedagógico, incluyendo cada uno de ellos a su vez hasta tres segmentos característicos y consecutivos del acto pedagógico. Por orden de aparición, en el primer segmento dominaría la actividad autoestructurante, en el segundo, tendrían lugar los mayores niveles de interacción entre la actividad autónoma y la intervención externa, y en el tercero sería la estrategia directiva la que asumiría el mayor protagonismo.

Es preciso matizar que la sucesión de segmentos a la que nos referimos asume una configuración a la vez lógica y cronológica, pues en cada uno de ellos la estrategia dominante se articula de manera recurrente con una o las otras dos mencionadas. Por ejemplo, en un segmento de actividad inicial de ciclo que se prevé, según queda indicado, como predominantemente autoestructurante, el profesor interviene automáticamente, y en la medida necesaria, en ayuda de un alumno que demuestra unas carencias especiales o requiere un asesoramiento específico. En realidad, el profesor está permanentemente en situación de alerta con el fin de adaptar su contribución a las contingencias de las sucesivas situaciones.

En cualquier caso, lo que por lo general sorprende realmente del presente planteamiento es que subvierte totalmente la pauta característica del modelo convencional de intervención pedagógica en la enseñanza de las L2 el cual incita invariablemente a iniciar el proceso general de aprendizaje por el mayor grado de directividad pedagógica y sistematización de contenidos para avanzar progresivamente hacia las cotas de mayor autonomía operativa y creatividad.

Conviene recordar sin embargo que el rasgo común del estilo de actividad directiva convencional consiste en arbitrar la distribución por fases de la totalidad del conocimiento, preseleccionándolo subjetivamente, conforme a unos criterios cartesianos para su transmisión ordenada mediante diversas técnicas de concienciación sistemática.

En la modalidad de intervención que propugnamos, la función de la estrategia directiva es básicamente reguladora, puesto que se limita a gestionar el apoyo a procesos de adquisición característicos señalados por la propia práctica. Se trata en concreto de aquellos procesos para los que las estrategias autónomas o relativamente autónomas demostraron previa y objetivamente su insuficiencia. De modo que las estrategias directivas y de construcción consciente del conocimiento que concebimos asumen en nuestra propuesta un papel, desde luego decisivo, aunque estricta y auténticamente compensatorio.

Por lo tanto, lo significativo del presente planteamiento es que a medida que avanza el proceso general de aprendizaje, lo que va en aumento no es la independencia operativa concedida a los procesos internos, sino la complejidad de la tarea, tanto si ésta resulta fundamentalmente autoestructurante como si es predominantemente dirigida.

Hay que precisar por otra parte que en nuestra alternativa, con los alumnos principiantes absolutos, toda la actividad pedagógica se apoya en una interrelación 
lingüística del profesor con el grupo grande, a la vez que de los alumnos entre sí, así como en un esfuerzo resolutivo colectivo. A los pocos meses, se inician las actividades por grupo y se evoluciona progresivamente hacia la individualización de las tareas. No obstante, el esquema que venimos describiendo es de aplicación regular prácticamente desde el principio del proceso de aprendizaje. Por tanto no sería correcto identificar mecánicamente por ejemplo individualización con independencia operativa, o trabajo con el grupo grande con procesos directivos, pues en las actividades solitarias los alumnos pueden recibir eventualmente la misma proporción de colaboración que la que permiten algunas actividades colectivas.

En el primer segmento, pretendemos como decimos dar la oportunidad a los aprendizajes más sencillos y asequibles, y que se verían obstaculizados por un exceso de conciencia y de sistematización, de resolverse mediante una actividad autoestructurante. En ese momento, los alumnos realizan una utilización libre de la lengua y del material de apoyo, y dan libre curso a su reflexión espontánea sobre el funcionamiento de la lengua y sobre los mejores modos de llevar a cabo la actividad encomendada. Paralelamente, la tarea fundamental del profesor consiste por una parte en proporcionar la información lingüística, organizativa y material que le es requerida para el desenvolvimiento eficaz en las situaciones creadas y, por otra, en estimular la iniciativa y el pensamiento.

La misión del profesor consiste también en observar, como es natural. Pues cada vez que los alumnos desarrollan una actividad autoestructurante propia del primer segmento, el profesor efectúa por su parte un seguimiento de los aspectos de las realizaciones de los alumnos que más le llaman la atención mediante los instrumentos correspondientes de observación y registro.

Es importante comprender que el profesor no procede a un seguimiento exhaustivo de la progresión de los alumnos ya que eso convertiría el proceso de evaluación en el centro absorbente de toda la estrategia de intervención. En efecto, para asegurar su mayor disponiblidad, el profesor no registra los aspectos de las realizaciones de los alumnos que pueden ser considerados como normales, sino estrictamente aquellos que evidencian una necesidad de ayuda, colaboración o refuerzo específicos y aquellos que denotan la existencia de unas facultades o aficiones destacables y por consiguiente la presencia de un potencial especial a tener en cuenta para futuros proyectos de actividades.

Esas operaciones permiten por tanto crear el mecanismo necesario para que los aprendizajes que se estimen decisivos y no superen satisfactoriamente el primer segmento, encuentren su segunda oportunidad en el segmento siguiente.

El segundo segmento se relaciona en una medida importante con tareas de corrección del escrito pues, por su carácter estático, esa modalidad se acomoda mejor a la observación pausada que la lengua oral. Supervisados los trabajos, el profesor no corrige propiamente, sino que señala los errores a la vez que muestra mediante un código sencillo un posible camino a seguir para facilitar una autocorrección asistida. Si la misma afecta a la producción lingüística, el camino señalado podrá ser el diccionario, o un material pedagógico específico especialmente confeccionado para los citados fines. Particularmente característicos del segundo segmento son también el diálogo pedagógico, la recomendación y la orientación en general.

El tercer segmento no aparece en realidad más que tras la realización de varios trabajos sucesivos. Las fichas individuales de seguimiento de la progresión del alumno permiten ir fijando su perfil pedagógico singular al evidenciar las dificultades particulares del grado tres que pueden afectar indistintamente a conceptos como la pronunciación, la ortografía, el uso de posesivos, la coherencia textual, la organización del trabajo o a problemas de tipo relacional. 
Posteriormente, se organiza un sistema adaptado de ayudas pertinentes al que se verán sometidos exclusivamente los alumnos que objetivamente lo precisan, y estrictamente para los procesos de desarrollo que manifiestamente reclaman esa suerte de apoyos y acomodaciones por parte de la intervención educativa.

De esta manera, y como pretendíamos, una interacción constructiva entre procesos autónomos y procesos dirigidos se encuentra asegurada ya que cada una de las estrategias de intervención comentada se limita a asumir la responsabilidad de compensar las insuficiencias resolutivas de la otra.

Para concluir, diremos que el sistema que postulamos está siendo aplicado desde hace varios años en diferentes experiencias de diverso rigor, dirigidas a adolescentes en un contexto de enseñanza reglada. Pese a ello, el planteamiento sigue reclamando sin duda abundantes análisis contrastivos, comprobaciones y complementaciones con otras interpretaciones teóricas y con las conclusiones de otros estudios empíricos.

En cualquier caso, el desafío que nos proponíamos recoger en este espacio era el de reunir en un marco conceptual común consistente unas ideas directrices de orden teórico-científico de indiscutida trascendencia para la práctica educativa, con la descripción suscinta de una experiencia pedagógica inspirada en gran medida por ellas.

Acrualmente, hemos encaminado nuestra reflexión en unas direcciones que creemos cruciales para el desbaratamiento definitivo de algunos de los prejuicios más anclados en las prácticas educativas convencionales. Guiándonos por la teoría de la toma de conciencia de Vygotski, intentamos contribuir a despejar el mundo de incógnitas que se esconde tras el concepto de conciencia de la lengua acotando los espacios semánticos que ocupan en primer lugar la idea de dominio operativo, que correspondería a la aplicación adecuada al uso espontáneo de la L2 de reglas del lenguaje distintas a las de L1, en segundo lugar la idea de dominio consciente, que supondría la capacidad para aplicar correcta y voluntariamente las reglas en un contexto de ejercicio o, en todo caso, al margen de cualquier contexto comunicativo, y finalmente la idea de dominio metalinguiistico que remitiría a la capacidad para referirse cabalmente al funcionamiento de las reglas. Nuestra hipótesis es que efectivamente ninguna de esas pericias presupone jamás la existencia de cualquier otra. Si diversos estudios empíricos llegaran a respaldar esa convicción es posible que, a la postre, los estilos de intervención dominantes dejaran de presuponer el milagro de la repercusión automática entre los distintos tipos de dominio de la lengua y en consecuencia de perseguirlos como fines en sí mismos. Sería indudablemente el anuncio del inicio de una etapa más racional para la pedagogía de las L2. 


\section{Criterios de pertinencia atribuibles a los actos pedagógicos en la enseñanza de una segunda lengua Concha Sanz Miguel$$
\text { CL\&E, 1994, 24, pp. 63-70 }
$$

Resumen: El aprendizaje de una segunda lengua está sobredeterminado por una pluralidad de factores de orden psicológico, sociológico, situacionales y relativos al pasado académico del alumno cuyo conocimiento sitúa al profesor en condiciones óptimas para sacar provecho de las motivaciones y potencialidades de desarrollo de los alumnos. Pero es preciso evitar someter al profesor a un ejercicio permanente de dilucidación de los mencionados factores de incidencia. Por otro lado, es obvio que la capacidad del sujeto para aprender excede $\longrightarrow$ en todo caso no coincide - con la capacidad de la intervención para prever, explicar, describir o incluso observar el flujo de la progresión del aprendizaje en toda su dimensión. Este planteamiento conduce a la necesidad de indagar unos criterios de pertinencia aplicables a los actos educativos entendida ésta como el atributo de todo aquello que favorece el aprendizaje o todo aquello que no lo obstaculiza. La conclusión es que el elemento clave de la pertinencia se ubica en la oportunidad y adecuación de la intervención en relación con la necesidad de ayuda que experimenta el alumno con motivo del acto; es decir que el acto resulta pertinente en la medida en que el alumno recibe ni más ni menos que el tipo de ayuda que necesita en el momento en que la necesita

Datos sobre la autora: Miembro del SELA (Seminario de Expresión Libre de Albacete). Centro Superior de Humanidades de Albacete

Dirección: Centro Superior de Humanidades. Universidad de Castilla-La Mancha. Plaza de la Universidad, 2. 20071 Albacete.

(C) PERMISOS PARA CITAR O REPRODUCIR EN OTRAS FUENTES: Se pueden citar libremente hasta 500 palabras. Para reproducir una porción de texto mayor, figuras o ilustraciones, se deberá pedir permiso por escrito a la revista, especificando el uso al que se destina el texto. En todos los casos, se deberá citar el copyright de $C L \& E$. En el caso de artículos o textos que hayan sido a su vez reproducidos en $C L \& E$ los interesados deberán dirigirse tanto a los detentadores del copyright original como a $C L \& E$, en el caso de que se quiera hacer uso de la traducción. FOTOCOPIAS: Para todo lo relacionado con el uso mediante fotocopia del material de esta revista, deberán dirigirse a: CEDRO, C/ José Marañón, 10, 3. Izda. Tel. 5941575 . Fax 4453567 